\title{
Sexual Dysfunction Seems to Trigger Depression in Female Multiple Sclerosis Patients
}

\author{
Katharina M. Hösl ${ }^{a}$ Martina Deutsch ${ }^{b}$ Ruihao Wang ${ }^{b}$ Sankanika Roy \\ Klemens Winder $^{\mathrm{b}}$ Günter Niklewski $^{\mathrm{a}}$ De-Hyung Lee ${ }^{\mathrm{b}}$ Ralf A. Linker ${ }^{\mathrm{b}}$ \\ Max J. Hilz, c \\ ${ }^{a}$ Department of Psychiatry and Psychotherapy, Paracelsus Medical University, Nuremberg, Germany; ${ }^{\mathrm{b}}$ Department \\ of Neurology, University of Erlangen-Nuremberg, Erlangen, Germany; ${ }^{C}$ Department of Neurology, Icahn School of \\ Medicine at Mount Sinai, New York, NY, USA
}

\section{Keywords}

Multiple sclerosis · Depression · Sexual dysfunction

\section{Abstract}

Background: In women with multiple sclerosis (MS), depression and sexual dysfunction (SD) are common. Whether SD promotes depression or vice versa remains unclear despite therapeutic relevance. Therefore, we aimed to assess whether SD more likely triggers depression or vice versa. Methods: In 83 female MS patients and 21 age-matched healthy women, we assessed depression, using the Beck Depression Inventory-V $(\mathrm{BDI}-\mathrm{V})$, and SD using the Female Sexual Function Index (FSFI). We diagnosed depression with BDI-V-scores $>35$ and SD with FSFI scores $<26.55$. We divided patients into groups with and without SD, with and without depression. Between groups, we compared prevalence of SD and depression (Fisher's-exact-test), age, MS-duration, MS-severity, BDI$\mathrm{V}-$, and FSFI scores (Mann-Whitney U-test; significance: $p<$ $0.05)$. Results: A total of $37 / 83$ MS patients and $1 / 21$ controls had SD; 28/83 patients and 3/21 controls had depression; $51.4 \%$ patients with SD but only $19.6 \%$ without SD had de-
() 2018 S. Karger AG, Basel pression $(p=0.003)$. SD was present in $67.9 \%$ depressed and $32.7 \%$ non-depressed patients. BDI-V-scores were higher in patients with SD than in patients without SD. FSFI scores were lower in depressed than non-depressed patients. Conclusion: In conclusion, SD was more common than depression. SD afflicted $67.9 \%$ depressed MS patients and was also more common in non-depressed MS patients than controls. SD may occur independently from depression while increased depressiveness seems linked to coexistent SD.

(c) 2018 S. Karger AG, Basel

\section{Introduction}

In multiple sclerosis (MS), depression and sexual dysfunction (SD) are common [1,2]. Depending on parameters such as patient age, disease severity, hospital or outpatient based settings, between 15.7 and $50 \%$ of the female MS patients suffer from depression [1, 3-7].

\section{K.M.H. and M.D. contributed equally to the manuscript.}

\section{KARGER}

E-Mail karger@karger.com www.karger.com/ene
Prof. Dr.med. Dr.med.habil. Max J. Hilz 
Depression may be due to psychological, psychosocial as well as organic pathologies arising from MS-specific brain lesions [1]. Depression affects cerebral neurophysiology [8], bodily functions [9] and psychosocial relationships [10], and may thus further SD in female MS patients.

Various studies show that the prevalence of SD among female MS patients ranges between 50 and 85\% [2, 1113]. Again, SD may be multifactorial. In addition to MSrelated cerebral or spinal lesions [11, 14-17], psychological or social problems [18], physical complications of MS, such as bladder and bowel dysfunction $[19,20]$ or spasticity, may give rise to $\mathrm{SD}[21,22]$.

In the general population, SD and depression are considered to be associated with each other due to a known bidirectional interdependence between depression and SD $[12,23]$. In female MS patients, previous studies also suggest an interdependence of SD, depression, disease severity, and duration $[12,13,24]$. However, it is so far unclear whether depression in female MS patients might be secondary to centrally induced SD, or whether SD might result from MS-related depression. This differentiation is clinically relevant since treatment of SD secondary to depression and treatment of depression secondary to $\mathrm{SD}$ may differ significantly [25], particularly since many antidepressant drugs aggravate SD $[25,26]$.

Several studies show that SD in MS patients is often associated with MS lesions in cerebral or spinal cord areas contributing to central pathways of sexual function [11, 14-17, 27]. Our group recently demonstrated associations between the site of MS-specific cerebral lesions and specific disorders of female sexual function, such as arousal, lubrication or orgasmic dysfunction $[14,15]$. Based on our previous studies [14,15,28], we hypothesize that primary SD [18] due to central MS lesions is a major contributing factor of depression in female MS patients.

We, therefore, evaluated whether there is evidence supporting the hypothesis that SD in female MS patients may cause depression or whether SD and depression are simply associated with each other due to a bidirectional interdependence between depression and SD [23, 25, 27].

\section{Patients and Methods}

\section{Patients with MS and Healthy Women}

A total of 83 women, aged 19-65 years (median age 36.2 years; interquartile range [IQR] 29.3-42.5 years), who had been diagnosed with MS according to the revised McDonald criteria [29] and 21 healthy, age-matched women (median age 33.2 years; IQR 28.250.3 years) participated in the questionnaire-based study. All
Table 1. Disease modifying therapy in our 83 female patients with multiple sclerosis

\begin{tabular}{lc}
\hline Medication & Number of patients, $n(\%)$ \\
\hline Interferon beta & $32 / 83(38.6)$ \\
Natalizumab & $19 / 83(22.9)$ \\
No medication & $16 / 83(19.3)$ \\
Fingolimod & $4 / 83(4.8)$ \\
Mitoxantrone & $3 / 83(3.6)$ \\
Glatiramer acetate & $2 / 83(2.4)$ \\
Daclizumab & $2 / 83(2.4)$ \\
Teriflunomid & $2 / 83(2.4)$ \\
Azathioprine & $1 / 83(1.2)$ \\
Dimethyl fumarate & $1 / 83(1.2)$ \\
Rituximab & $1 / 83(1.2)$ \\
\hline
\end{tabular}

patients were treated at the MS center of the Department of Neurology at the University Erlangen-Nuremberg, Erlangen, Germany. Of which, 76 patients had relapsing-remitting MS, 6 patients had secondary progressive MS, 1 patient had primary progressive MS. A total of 32 patients received interferon beta, 19 patients were treated with Natalizumab, 16 patients received no therapy, and 16 patients received Fingolimod, Mitoxantrone, Glatiramer acetate, or other disease modifying therapies shown in Table 1 .

Women younger than 19 years or older than 65 years and patients with other neurological disorders and diseases or on medication possibly interfering with autonomic cardiovascular modulation, including antidepressants, were excluded from the study. None of the participants had received any antidepressant therapy or had been treated because of SD. The study was approved by the Ethics Committee of the University of Erlangen-Nuremberg, and all participants had given written informed consent according to the Declaration of Helsinki after a detailed explanation of the study purpose and contents. All participants underwent physical and neurological examinations. In the patients, MS severity was scored according to the Expanded Disability Status Scale (EDSS) ranging from 0 to 10. Scores from 0 to 4.5 indicate that patients still have the full capability of walking; higher scores indicate more severe handicap [30].

\section{Assessment of SD}

To assess prevalence and severity of SD in healthy participants and MS patients, we applied the Female Sexual Function Index (FSFI), a widely used standardized 19-item self-report questionnaire validated for the general population [31]. The FSFI covers the 6 sexual domains: desire, arousal, lubrication, orgasm, satisfaction, and pain [31]. Desire is evaluated by 2 questions, arousal by 4 questions, lubrication by 4 questions, orgasm by 3 questions, satisfaction by 3 questions, and pain by 3 questions. Each question can be answered with scores from 0 or 1 to 5 , with higher values indicating better function and less pain during sexual activity or intercourse. The total score of each of the 6 domains is adjusted by a validated correction factor that assigns a maximum score of 6 to each of the 6 domains. Thus, the total maximum FSFI score is 36 . The lower the scores, the more severe is the female SD or the dysfunction in one of the 6 domains [31]. Female SD is diagnosed when the total FSFI score is below 26.55 points [32]. 
Table 2. Prevalence of SD and depression in 83 female MS patients and 21 healthy women

\begin{tabular}{|c|c|c|c|c|c|c|}
\hline \multirow[t]{2}{*}{ Parameters } & \multirow[t]{2}{*}{$\begin{array}{l}\text { MS patients with } \\
\text { SD }(n=37)[1]\end{array}$} & \multirow[t]{2}{*}{$\begin{array}{l}\text { MS patients without } \\
\text { SD }(n=46)[2]\end{array}$} & \multirow[t]{2}{*}{$\begin{array}{l}\text { Healthy women } \\
(n=21)[3]\end{array}$} & \multicolumn{3}{|c|}{$\begin{array}{l}\text { Comparison between groups } \\
\text { Fisher's exact test } p \text { value }\end{array}$} \\
\hline & & & & [1] vs. [2] & [1] vs. [3] & [2] vs. [3] \\
\hline Depression, $n(\%)$ & $19 / 37(51.4)$ & 9/46 (19.6) & $3 / 21(14.3)$ & 0.003 & 0.011 & 0.738 \\
\hline No depression, $n(\%)$ & $18 / 37(48.6)$ & $37 / 46(80.4)$ & $18 / 21(85.7)$ & 0.003 & 0.011 & 0.738 \\
\hline Parameters & $\begin{array}{l}\text { Depressed } \\
\text { MS patients } \\
(n=28)[1]\end{array}$ & $\begin{array}{l}\text { Non-depressed } \\
\text { MS patients } \\
(n=55)[2]\end{array}$ & $\begin{array}{l}\text { Healthy } \\
\text { women } \\
(n=21)[3]\end{array}$ & [1] vs. [2] & [1] vs. [3] & [2] vs. [3] \\
\hline $\mathrm{SD}, n(\%)$ & $19 / 28(67.9)$ & $18 / 55(32.7)$ & $1 / 21(4.8)$ & 0.003 & $<0.001$ & 0.016 \\
\hline No SD, $n(\%)$ & $9 / 28(32.1)$ & $37 / 55(67.3)$ & $20 / 21(95.2)$ & 0.003 & $<0.001$ & 0.016 \\
\hline
\end{tabular}

MS, multiple sclerosis; SD, sexual dysfunction.

To evaluate depressiveness, all participants were asked to fill out an abridged 20-item version of the Beck Depression Inventory (BDI-V) [33]. The 20 questions evaluate the presence and severity of depressive symptoms based on depression criteria of the Diagnostic and Statistical Manual of Disorders (DSM-IV). Each question can be scored from 0 to 5 , with higher values indicating more severe symptoms and a sum scores above 35 indicating clinically relevant depression with a $90 \%$ probability [34].

Based on FSFI scores below or at and above 26.55, we divided patients into groups with SD and without SD. Based on BDI-V scores above or at and below 35, we assigned patients to the groups with or without depression. Finally, we also assigned patients to a group meeting criteria of both SD and depression. Between the groups, we compared the prevalence of SD and depression, as well as severity of depressiveness, as assessed by BDI-V scores, and of SD, as assessed by FSFI scores; in addition, we compared age, disease duration and EDSS scores between the groups.

In all 83 patients and in the above subgroups, we calculated correlations between FSFI scores, BDI-V scores, patient age, disease duration and severity to further explore whether there are associations supporting the hypothesis that $\mathrm{SD}$ might trigger depression or vice versa.

\section{Statistical Analysis}

To test data for normal distribution, we used the Shapiro-Wilk test. Not normally distributed data are presented as median and IQR; normally distributed data are presented as mean and standard deviation. To compare the prevalence of categorical data, such as prevalence of depression or SD, we used the Fisher's exact test. To compare scores and data between healthy participants and the patient groups without and with depression, and data between healthy participants and patient groups without and with SD, we used the Kruskal-Wallis test for not normally distributed data and analysis of variance for normally distributed data. To compare data between 2 of the 3 groups, we used the Mann-Whitney U-test for not normally distributed data and $t$ tests for normally distributed data.
To calculate correlations, we used the Spearman rank correlation test for not normally distributed data. Statistics were calculated with a commercially available statistical program (IBM SPSS Statistics for Windows, Version 20.0. Armonk, NY, USA). Significance was assumed for $p<0.05$.

\section{Results}

All participants filled out questionnaires. The age did not differ between healthy participants (median age 36.2 years, IQR 29.3-42.5 years) and patients (median age 33.2 years, IQR 28.2-50.3 years; Mann-Whitney U-test: $p$ > $0.05)$.

\section{Prevalence of SD and Depression in $83 \mathrm{MS}$ Patients and 21 Controls (Table 2)}

SD was prevalent in 37 of the 83 MS patients (44.6\%) and 1 of the 21 healthy women (4.8\%; Fisher's exact test: $p<0.001)$. Depression was prevalent in 28 of the 83 patients (33.7\%) and in 3 of the 21 healthy participants (14.3 $\%$ ), one of whom also had SD (Fisher's exact test: $p=$ 0.066 ).

A total of 19 of the 37 (51.4\%) patients with SD (i.e., $22.9 \%$ of all 83 patients) also had depression while only nine of the 46 (19.6\%) patients without SD had depression (Fisher's exact test: $p=0.003$ ). Prevalence of depression was also higher in the 37 patients with SD than in healthy women but did not differ between the $46 \mathrm{MS}$ patients without SD and healthy women.

A total of 19 of the 28 patients with depression also had SD $(67.8 \%)$. And 18 of the 55 patients (32.7\%) without depression had SD (2-sided Fisher's exact test: $p=0.005)$.
Eur Neurol 2018;80:34-41 DOI: $10.1159 / 000492126$
Hösl/Deutsch/Wang/Roy/Winder/ Niklewski/Lee/Linker/Hilz 


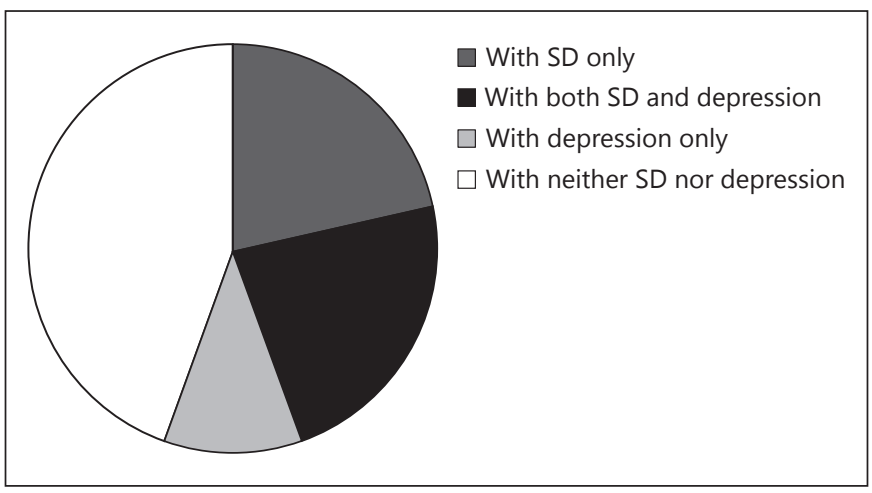

Fig. 1. Prevalence of sexual dysfunction and depression in $83 \mathrm{fe}-$ male patients with multiple sclerosis (MS). Among 83 female MS patients, 18 patients had sexual dysfunction but no depression (gray shade), 19 patients had both sexual dysfunction and depression (black shade), 9 patients had depression but no sexual dysfunction (light gray), and 37 patients neither had sexual dysfunction nor depression.

SD was more prevalent among the 28 MS patients with depression as well as the 55 patients without depression than among healthy women (Fig. 1).

\section{Severity of SD and Depressiveness in 37 Patients}

with $S D, 46$ Patients without $S D$, and in

21 Controls (Table 3a)

Age did not differ between the 37 patients with SD, the 46 patients without SD, and the 21 healthy participants, and disease duration and disability (as measured by EDSS) did not differ between the patients with and without SD. However, SD was more severe, that is, FSFI scores were significantly lower in the 37 patients with SD than the 46 patients without SD or the 21 healthy participants while FSFI scores did not differ between patients without SD and healthy participants. BDI-V scores were also higher in the 37 patients with SD (median 36; IQR 25.5-49.5) than in the 46 patients without SD (median 20; IQR 12-29) and the 21 healthy participants (median 18; IQR 10.5-28.5). However, BDI-V scores did not differ between patients without SD and the healthy participants.

\section{Severity of Depressiveness and SD in 28 Depressed,}

55 Non-Depressed Patients, and in

21 Controls (Table 3b)

Again, age did not differ between the 28 depressed, 55 non-depressed patients, and 21 controls, and disease duration and disability as measured by EDSS did not differ between depressed and non-depressed patients.
As expected, BDI-V scores were higher in the 28 depressed patients than in the 55 non-depressed patients and the 21 controls while BDI-V scores did not differ between non-depressed patients and controls. In the 28 depressed patients, FSFI scores were also significantly lower (median: 21.0: IQR 5.9-27.8) than in the 55 non-depressed MS patients (median: 29.6; IQR: 24.4-32.6; $p=$ 0.001) or the controls (median 31; IQR 29.3-33.5). Again, FSFI values did not differ significantly between non-depressed patients and controls.

\section{Correlations between Age, Disease Duration, Disease}

Severity, Depressiveness, and SD (Table 4)

FSFI scores inversely correlated with age in the group comprising all patients and in the subgroups with SD and/or depression. In contrast, FSFI scores only correlated inversely with disease severity in the group comprising all patients and the subgroup with depression. Similarly, FSFI scores correlated inversely with BDI-V scores only in the group comprising all patients. Otherwise, BDI-V scores correlated only with disease severity in the group comprising all patients.

\section{Discussion}

In our patients, $\mathrm{SD}$ and depression were not only more prevalent than in the controls but patients were more frequently afflicted by SD (44.6\%) than depression (33.7\%). Our data again confirm the previously described association between SD and depression in female MS patients $[12,23]$ and show a similar prevalence of SD and depression in our patients as in previous studies which suggest that SD prevalence may vary considerably and affect 40 $80 \%$ of female MS patients [2, 12, 21, 24, 35]. Similarly, prevalence of depression varied in previous studies with patient age, MS severity, hospital or out-patient based study-setting, and ranged from 15.7 to $50 \%$ [1, 3-7].

In our patients, MS severity also affected the severity of SD and depression, as shown by the correlation between EDSS scores and FSFI as well as BDI-V scores (Table 4) $[20,24]$.

However, in contrast to previous studies, we not only saw an increased prevalence and close interdependence of depression and SD in the female MS patients $[1,3,4]$, but also the differentiation of patients into subgroups with and without SD as well as subgroups with and without depression. This strongly suggests that SD is more closely linked to depression than vice versa ( $\mathrm{Ta}$ ble 2). 
Table 3.

a. Age, disease duration, EDSS, BDI-V-scores, and FSFI scores in 37 MS patients with sexual dysfunction defined by FSFI scores below 26.55, in $46 \mathrm{MS}$ patients without SD defined by FSFI scores at or above 26.55, and in 21 healthy women

\begin{tabular}{|c|c|c|c|c|c|c|}
\hline \multirow[t]{2}{*}{ Parameters } & \multirow{2}{*}{$\begin{array}{l}\text { MS patients } \\
\text { with SD } \\
(n=37)[1]\end{array}$} & \multirow{2}{*}{$\begin{array}{l}\text { MS patients } \\
\text { without SD } \\
(n=46)[2]\end{array}$} & \multirow{2}{*}{$\begin{array}{l}\text { Healthy } \\
\text { women } \\
(n=21)[3]\end{array}$} & \multicolumn{3}{|c|}{$\begin{array}{l}\text { Comparison between groups }{ }^{*, \dagger} \\
p \text { value }\end{array}$} \\
\hline & & & & [1] vs. [2] & [1] vs. [3] & [2] vs. [3] \\
\hline Age, years & $39.9(31.5-43.9)$ & $34.6(27.3-42.0)$ & $33.2(28.2-50.3)$ & 0.162 & & \\
\hline MS, months & $58.5(10.7-112.5)$ & $43.0(9.0-94.5)$ & NA & 0.651 & NA & NA \\
\hline EDSS & $3(1.5-6)$ & $2(1-4)$ & NA & 0.111 & NA & NA \\
\hline BDI-V & $36(25.5-49.5)$ & $20(12-29)$ & $18(10.5-28.5)$ & $<0.001$ & $<0.001$ & 0.620 \\
\hline FSFI & $16.4(4.7-23.4)$ & $31.4(29.2-33.2)$ & $31(29.3-33.5)$ & 0.001 & $<0.001$ & 0.962 \\
\hline
\end{tabular}

b. Age, disease duration, EDSS, BDI-V-scores, and FSFI scores in 28 depressed MS patients defined by BDI-V scores above 35 , in 55 non-depressed MS patients, and in 21 healthy women

\begin{tabular}{|c|c|c|c|c|c|c|}
\hline \multirow[t]{2}{*}{ Parameters } & \multirow{2}{*}{$\begin{array}{l}\text { Depressed } \\
\text { MS patients } \\
(n=28)[1]\end{array}$} & \multirow{2}{*}{$\begin{array}{l}\text { Non-depressed } \\
\text { MS patients } \\
(n=55)[2]\end{array}$} & \multirow{2}{*}{$\begin{array}{l}\text { Healthy } \\
\text { women } \\
(n=21)[3]\end{array}$} & \multicolumn{3}{|c|}{$\begin{array}{l}\text { Comparison between groups*, } \dagger \\
p \text { value }\end{array}$} \\
\hline & & & & [1] vs. [2] & [1] vs. [3] & [2] vs. [3] \\
\hline Age, years & $33.3(28.7-41.2)$ & $38.5(29.3-43.0)$ & $33.2(28.2-50.3)$ & 0.471 & & \\
\hline MS duration, months & $43(10-93)$ & $52.5(9.8-129.8)$ & $\mathrm{NA}$ & 0.829 & NA & NA \\
\hline EDSS & $3(1.5-6)$ & $2(1-4)$ & NA & 0.154 & NA & NA \\
\hline BDI-V & $49.5(42.3-58.5)$ & $20(12-27)$ & $18(10.5-28.5)$ & $<0.001$ & $<0.001$ & 0.862 \\
\hline FSFI & $21.0(5.9-27.8)$ & $29.6(24.4-32.6)$ & $31(29.3-33.5)$ & 0.001 & $<0.001$ & 0.073 \\
\hline
\end{tabular}

Data are expressed as median values and interquartile ranges. Significant differences were indicated in bold.

* Kruskal-Wallis $\mathrm{H}$ test was used for the comparison between the 3 groups.

† Mann-Whitney U test was used for the post-hoc comparison between 2 groups.

BDI, Beck depression inventory; EDSS, expanded disability status scale; FSFI, female sexual function index; NA, not available.

Table 4. Correlation of FSFI scores with age, disease duration, EDSS, and BDI-V scores and correlation of BDI-V scores with age, disease duration, and EDSS scores in

A. 83 female MS patients

B. 37 female MS patients with sexual dysfunction defined by FSFI scores below 26.55, and

C. 28 female MS patients with depression defined by BDI-V scores above 35

D. 19 female MS patients with both sexual dysfunction and depression

\begin{tabular}{|c|c|c|c|c|c|c|c|c|}
\hline & \multicolumn{2}{|l|}{ Age } & \multicolumn{2}{|c|}{ Disease duration } & \multicolumn{2}{|l|}{ EDSS } & \multicolumn{2}{|l|}{ BDI-V } \\
\hline A. $(n=83)$ & -0.271 & 0.007 & -0.026 & 0.825 & -0.243 & 0.031 & -0.489 & 0.000 \\
\hline B. $(n=37)$ & -0.471 & 0.003 & 0.094 & 0.596 & -0.378 & 0.025 & -0.172 & 0.310 \\
\hline C. $(n=28)$ & -0.478 & 0.010 & -0.047 & 0.816 & -0.062 & 0.757 & 0.054 & 0.786 \\
\hline A. $(n=83)$ & -0.070 & 0.527 & 0.056 & 0.635 & 0.233 & 0.039 & $\mathrm{~N} / \mathrm{A}$ & $\mathrm{N} / \mathrm{A}$ \\
\hline B. $(n=37)$ & -0.172 & 0.308 & -0.078 & 0.660 & 0.141 & 0.419 & $\mathrm{~N} / \mathrm{A}$ & $\mathrm{N} / \mathrm{A}$ \\
\hline C. $(n=28)$ & -0.084 & 0.671 & -0.203 & 0.310 & 0.082 & 0.685 & $\mathrm{~N} / \mathrm{A}$ & $\mathrm{N} / \mathrm{A}$ \\
\hline D. $(n=19)$ & 0.218 & 0.370 & -0.043 & 0.861 & 0.119 & 0.628 & $\mathrm{~N} / \mathrm{A}$ & $\mathrm{N} / \mathrm{A}$ \\
\hline
\end{tabular}

FSFI, female sexual function index; BDI, Beck depression inventory; EDSS, expanded disability status scale; rho, Spearman rank correlation rho; N/A, not available. Significant differences were indicated in bold. 
A total of $51.4 \%$ of our patients with SD also suffered from depression (Table 2), and depressiveness was more severe in patients with SD than in those without SD (Table 3a). In fact, in patients with normal sexual function depressiveness did not differ from depressiveness in controls (Tables 2, 3b). In contrast, SD was not only more prevalent in the patient subgroup without depression than in the control group ( 32.7 vs. $4.8 \%$; Table 2 ) but also more severe (2-sided Fisher's exact test: $p=0.016$; Table $3 \mathrm{~b})$. In the subgroup of depressed patients, SD was even more prevalent $(67.9 \%)$ and again more severe than in the subgroup without depression (Tables 2, 3b).

While FSFI scores reflecting sexual function correlated negatively with patient age, BDI-V scores reflecting depressiveness neither correlated with patient age nor disease duration but only with disease severity. The age-related decline in sexual function can be explained by the age-related decrease in sexual function in the general population $[25,27,36]$. However, the link between deteriorating sexual function, that is, decreasing FSFI scores and increasing depressiveness, that is, increasing BDI-V scores was only significant for the entire group of our 83 patients but not for the subgroups of depressed and non-depressed patients, nor for patients with and without SD (Table 4). This might be due to an inadequate sample size of the subgroups. There was no correlation between FSFI scores and BDI-Vscores within the subgroup of the 28 depressed patients or within the subgroup of the 37 patients with SD. This might also suggest that the severity of 1 disorder does not necessarily influence the severity of the other disorder despite the above mentioned bidirectional interdependence between depression and SD $[12,23]$. Theoretically, SD and depression might trigger each other without mutually influencing the severity of the other disorder.

In summary, our data not only show that SD is more common than depression among female MS patients but show that depression without SD (9/46 patients) is neither more common nor more pronounced among our MS patients than among our healthy controls (3/21 women). In contrast, SD is not only closely linked to depressiveness and afflicts $67.8 \%$ of our depressed patients, but also occurred significantly more often in MS patients who have no depression than in our healthy controls (Tables $2,3)$. While SD occurs together with depression in most of our patients, SD may also occur without concomitant depression (Table 2). In contrast, prevalence and severity of depressiveness (Tables 2, 3a, 3b) seem to be associated with coexistent SD.

Sexual Dysfunction and Depression in Female MS Patients
As mentioned earlier, the pathophysiology of SD and depression in MS may be multifactorial $[1,23]$ and, similar to the general population, may be based on a bidirectional association between depression and SD [23]: individuals with depression have an increased risk of developing $\mathrm{SD}$, while patients with $\mathrm{SD}$ are at an increased risk of depression [23]. In MS patients, not only psychological, somatic or social implications of MS contribute to depression [1] and SD [23], but also MS-specific lesions, for example, in projection areas of the basal limbic system [37] give rise to depressive symptoms such as anhedonia or depressed affect [1,37-39]. In 109 women with MS, Gold et al. [38] found associations between depression and reduced thickness of the right hippocampus. In 92 MS patients, Feinstein et al. [39] observed that increased lesion volume in the right medial inferior frontal region was associated with depression $[1,39]$. Berg et al. [37] reported associations between depression and increased MS lesion load in the right temporal lobe in 78 MS patients.

Similarly, central MS lesions are associated with SD $[11,16,17]$. Barak et al. [11] described associations of anorgasmia with brainstem lesions, lesions of the corticospinal tract and also with the total lesion load. In female and male MS patients, Zivadinov et al. [16] and Zorzon et al. [17] found associations between SD and pontine lesions. Our group recently showed associations between the site of MS-specific cerebral lesions and female SD [14, 15]. We found associations between right occipital lesions and impaired arousal and between left insular lesions and decreased lubrication [14]. In another study, we found that MS lesions in left temporal periventricular and right visual association areas compromise orgasmic function [15].

Based on our data, we assume that MS-specific lesions may contribute to SD which might entail depression, either due to lesion sites that yield both SD and depression, or due to secondary and psychological sequelae of SD [25, 27].

Our sample size was rather small due to our stringent criteria excluding participants with other diseases or on any therapy possibly influencing mood or sexual function. Still, our data suggest that SD is more common than depression among female MS patients. Moreover, the findings suggest that SD probably even triggers depression while the effect of depression on SD seems less prominent.

While we did not analyze the associations between specific central lesion sites in magnetic resonance images and depression or SD in this study, our previous studies 
indicate a prominent influence of central MS lesions on sexual function $[14,15,28]$. Although EDSS scores were below 4 in most of our patients, $44.6 \%$ of patients fulfilled the SD criteria and $33.7 \%$ met the criteria for depression. As expected, there was a close link between SD and depressiveness in the entire group of the 83 patients confirming the mutual interdependence of both disorders $[12,23]$.

However, only, those patients who had SD also had an increased prevalence and severity of depression (Table 2). In contrast, $\mathrm{SD}$ was more prevalent in the subgroups, regardless of coexistent depression, than in controls (Table 2 ). Thus, in our MS patients SD is not only more common than depression but our data suggest that SD may be a causative component of depression.

\section{Acknowledgement}

The study was partially funded by Bayer Vital GmbH, Germany.

\section{Statement of Ethics}

The Ethics Committee of the University of Erlangen-Nuremberg, Germany had approved the study, and written informed consent was obtained from all participants according to the Declaration of Helsinki (2000) of the World Medical Association.

\section{Disclosure Statement}

On behalf of all authors, the corresponding author states that there is no conflict of interest.

\section{References}

1 Feinstein A, Magalhaes S, Richard JF, Audet B, Moore C: The link between multiple sclerosis and depression. Nat Rev Neurol 2014;10: 507-517.

2 Lew-Starowicz M, Rola R: Prevalence of sexual dysfunctions among women with multiple sclerosis. Sex Disabil 2013;31:141-153.

3 Sadovnick AD, Remick RA, Allen J, et al: Depression and multiple sclerosis. Neurology 1996;46:628-632.

4 Patten SB, Beck CA, Williams JV, Barbui C, Metz LM: Major depression in multiple sclerosis: a population-based perspective. Neurology 2003;61:1524-1527.

5 Chwastiak L, Ehde DM, Gibbons LE, Sullivan M, Bowen JD, Kraft GH: Depressive symptoms and severity of illness in multiple sclerosis: Epidemiologic study of a large community sample. Am J Psychiatry 2002;159:18621868.

6 Marrie RA, Fisk JD, Yu BN, et al: Mental comorbidity and multiple sclerosis: Validating administrative data to support population-based surveillance. BMC Neurol 2013; 13:16.

7 Jones KH, Ford DV, Jones PA, et al: A largescale study of anxiety and depression in people with multiple sclerosis: a survey via the web portal of the UK MS register. PLoS One 2012;7:e41910.

8 Riccelli R, Passamonti L, Cerasa A, et al: Individual differences in depression are associated with abnormal function of the limbic system in multiple sclerosis patients. Mult Scler 2016;22:1094-1105.

9 Penninx BW, Leveille S, Ferrucci L, van Eijk JT, Guralnik JM: Exploring the effect of depression on physical disability: longitudinal evidence from the established populations for epidemiologic studies of the elderly. Am J Public Health 1999;89:1346-1352.
10 Hibbard MR, Ashman TA, Spielman LA, Chun D, Charatz HJ, Melvin S: Relationship between depression and psychosocial functioning after traumatic brain injury. Arch Phys Med Rehabil 2004;85(4 suppl 2):S43-S53.

11 Barak Y, Achiron A, Elizur A, Gabbay U, Noy $S$, Sarova-Pinhas I: Sexual dysfunction in relapsing-remitting multiple sclerosis: magnetic resonance imaging, clinical, and psychological correlates. J Psychiatry Neurosci 1996;21: 255-258.

12 Mohammadi K, Rahnama P, Mohseni SM, Sahraian MA, Montazeri A: Determinants of sexual dysfunction in women with multiple sclerosis. BMC Neurol 2013;13:83.

13 Lew-Starowicz M, Rola R: Correlates of sexual function in male and female patients with multiple sclerosis. J Sex Med 2014a;11:21722180.

14 Winder K, Linker RA, Seifert F, et al: Neuroanatomic correlates of female sexual dysfunction in multiple sclerosis. Ann Neurol 2016; 80:490-498.

15 Winder K, Seifert F, Koehn J, et al: Site and size of multiple sclerosis lesions predict enhanced or decreased female orgasmic function. J Neurol 2015;262:2731-2738.

16 Zivadinov R, Zorzon M, Locatelli L, et al: Sexual dysfunction in multiple sclerosis: a MRI, neurophysiological and urodynamic study. J Neurol Sci 2003;210:73-76.

17 Zorzon M, Zivadinov R, Locatelli L, et al: Correlation of sexual dysfunction and brain magnetic resonance imaging in multiple sclerosis. Mult Scler 2003;9:108-110.

18 Sanders AS, Folye FW, LaRocca NG, Zemon $\mathrm{V}$ : The multiple sclerosis intimacy and sexuality questionnaire-19 (MSISQ-19). Sex Disabil 2000;18:3-26

19 Fragala E, Privitera S, Giardina R, et al: Determinants of sexual impairment in multiple sclerosis in male and female patients with lower urinary tract dysfunction: results from an italian cross-sectional study. J Sex Med 2014;11:2406-2413.

20 Hulter BM, Lundberg PO: Sexual function in women with advanced multiple sclerosis. J Neurol Neurosurg Psychiatry 1995;59:8386.

21 Cordeau D, Courtois F: Sexual disorders in women with MS: assessment and management. Ann Phys Rehabil Med 2014;57:337347.

22 Orasanu B, Frasure H, Wyman A, Mahajan ST: Sexual dysfunction in patients with multiple sclerosis. Mult Scler Relat Disord 2013;2: 117-123.

23 Atlantis E, Sullivan T: Bidirectional association between depression and sexual dysfunction: a systematic review and meta-analysis. J Sex Med 2012;9:1497-1507.

24 Demirkiran M, Sarica Y, Uguz S, Yerdelen D, Aslan K: Multiple sclerosis patients with and without sexual dysfunction: are there any differences? Mult Scler 2006;12:209-214.

25 Hilz MJ: Female and male sexual dysfunction; in Low PA, Benarroch EE (eds): Clinical Autonomic Disorders (ed 3). Philadelphia, Lippincott Williams \& Wilkins, 2008, pp 657711.

26 Lorenz T, Rullo J, Faubion S: Antidepressantinduced female sexual dysfunction. Mayo Clin Proc 2016;91:1280-1286.

27 Hilz MJ: Physiology and pathophysiology of female sexual function; in Robertson D, Biaggioni I, Burnstock G (eds): Primer of the Autonomic Nervous System. Oxford, Academic Press, 2012, pp 235-238.

28 Koehn J, Crodel C, Deutsch M, et al: Erectile dysfunction (ED) after ischemic stroke: Association between prevalence and site of lesion. Clin Auton Res 2015;25:357-365. 
29 Polman CH, Reingold SC, Banwell B, et al: Diagnostic criteria for multiple sclerosis: 2010 revisions to the mcdonald criteria. Ann Neurol 2011;69:292-302.

30 Kurtzke JF: Rating neurologic impairment in multiple sclerosis: an expanded disability status scale (EDSS). Neurology 1983;33:14441452.

31 Rosen R, Brown C, Heiman J, et al: The female sexual function index (FSFI): a multidimensional self-report instrument for the assessment of female sexual function. J Sex Marital Ther 2000;26:191-208.

32 Wiegel M, Meston C, Rosen R: The female sexual function index (FSFI): cross-validation and development of clinical cutoff scores. J Sex Marital Ther 2005;31:1-20.

33 Schmitt JM, Maes J: Vorschlag zur vereinfachung des beck-depressions-inventars (BDI). Diagnostica 2000;46:38-46.

34 Schmitt M, Altstötter-Gleich C, Hinz A, Maes J, Brähler E: Normwerte für das vereinfachte beck-depressions-inventar (BDI-v) in der allgemeinbevölkerung. Diagnostica 2006;52: 51-59.

35 Foley FW, Sanders A: Sexuality, multiple sclerosis and women. Mult Scler Manage 1997;1: $1-9$.

36 Hayes R, Dennerstein L: The impact of aging on sexual function and sexual dysfunction in women: a review of population-based studies. J Sex Med 2005;2:317-330.

37 Berg D, Supprian T, Thomae J, et al: Lesion pattern in patients with multiple sclerosis and depression. Mult Scler 2000;6:156-162.

38 Gold SM, O'Connor MF, Gill R, et al.: Detection of altered hippocampal morphology in multiple sclerosis-associated depression using automated surface mesh modeling. Hum Brain Mapp 2014;35:30-37.

39 Feinstein A, O'Connor P, Akbar N, Moradzadeh L, Scott CJ, Lobaugh NJ: Diffusion tensor imaging abnormalities in depressed multiple sclerosis patients. Mult Scler 2010;16:189196. 